\title{
Gain dependence of the phase-shift spectra measured in coherent Brillouin optical time-domain analysis sensors
}

\author{
Jon Mariñelarena, Javier Urricelqui, Alayn Loayssa, Member, IEEE
}

\begin{abstract}
We report on the effects of large pump pulse powers on Brillouin optical time-domain analysis (BOTDA) sensors based on phase-modulated probe wave and coherent self-heterodyne detection. These sensors are particularly suitable to perform dynamic strain and temperature measurements because the RF signal that is obtained when the probe wave is detected has a phase-shift spectrum that is independent to first order of the Brillouin gain. Therefore, a fixed optical frequency separation between pump and probe wave can be deployed and use the stable RF phase-shift spectrum to obtain the Brillouin frequency shift (BFS) from measured changes in the probe RF phase-shift. However, in this work, it is found that there is a narrowing of the RF phase-shift spectrum that depends on the Brillouin gain. This effect becomes significant when very high power pulses are used and the resultant large gain induces a narrowing of the RF phase-shift spectrum. This narrowing leads to a BFS measurement error when the sensor is configured for dynamic measurements. We analyze, theoretically and experimentally, the origins and the magnitude of the narrowing of RF phase-shift spectra for high pump pulses in a coherent BOTDA sensor. Furthermore, this spectral shape change is compared to the broadening of the gain spectrum that has been recently discovered in conventional direct-detection BOTDA sensors, which is linearly dependent on the pulse peak power injected to the fiber, finding that the spectral shape change is less significant in coherent BOTDA sensors. Finally, we quantify the BFS measurement error that it can induce and find the trade-offs to keep it below a certain threshold. It is found that, from a practical point of view, this effect is significant for short fibers, where nonlinear effects are negligible and large pump pulses can be used.
\end{abstract}

Index Terms-Brillouin optical time-domain analysis, Brillouin scattering, coherent sensors, dynamic sensing.

\section{INTRODUCTION}

$\mathbf{N}$ EW techniques have been proposed in the last years to extend the Brillouin optical time-domain analysis (BOTDA) sensor to dynamic measurements [1]-[5]. The objective is to be able to measure fast strain and temperature variations with high precision and resolution, satisfying the

Manuscript received YYY, YYYY; revised YYY, YYYY. This work was supported by the Universidad Pública de Navarra, Feder funds and Spanish Ministerio de Economía y Competitividad through the project TEC201347264-C2-2-R.

The authors are with the Department of Electrical and Electronic Engineering, Universidad Pública de Navarra, 31006 Pamplona, Spain (e-mail: jon.marinelarena@unavarra.es; javier.urricelqui@unavarra.es; alayn.loayssa@unavarra.es.

Copyright (c) 2015 IEEE. Personal use of this material is permitted However, permission to use this material for any other purposes must be obtained from the IEEE by sending a request to pubs-permissions@ieee.org. increasing demand for distributed fiber sensors applied to structural health monitoring applications.

The first dynamic measurement techniques were based on the frequency discriminator principle, the so-called slope-assisted BOTDA [1], [2], [6]. This technique is based on fixing the pump and probe wavelength difference so that the probe lies in the slope of the Brillouin gain spectrum. Then, changes of the Brillouin frequency shift (BFS) are converted to changes of the detected probe power by the slope of the Brillouin gain spectrum (BGS) [1], [2], [6]. However, recently, a previously unknown effect in stimulated Brillouin scattering (SBS) interaction has been discovered, by which the linewidth of the BGS has been found to depend on the pump pulse power [7], [8]. This unexpected finding means that BFS measurement errors can appear in techniques such as the slope-assisted BOTDA that rely on the shape of the BGS [9]. This effect is just signifcant in short fibers, where nonlinear effects are negligible and thus, high power pulses can be deployed.

We have recently proposed a dynamic BOTDA sensor tolerant to Brillouin gain changes in the sensing fiber [3]. This technique is based on the measurement of the phase-shift spectra of the RF signal that results from the coherent self-heterodyne detection of a phase-modulated probe wave. The RF phase-shift is found to be dependent just on the BFS experienced by the probe wave, but not on the pump pulse power, for the range of Brillouin gains normally used in BOTDA sensors. Therefore, a fixed optical frequency separation between pump and probe waves can be deployed and use the stable RF phase-shift spectrum to obtain the BFS from measured changes in the probe RF phase-shift.

In this paper, we study the Brillouin gain dependence of the RF phase-shift spectrum that is measured in this coherent BOTDA system when higher pump pulse powers than usual are deployed. This is important in order to quantify the BFS measurement error that can be induced by changes of the RF phase-shift spectrum shape. We perform this investigation employing large pump power and Brillouin gain because no significant shape change was observed when moderate gain was used in our previous investigation. In addition to our study of shape alterations in coherent BOTDA, we also independently confirm the existence of the Brillouin gain linewidth dependence on the power pump pulses in conventional direct-detection BOTDA sensors [7], [8], which linearly broadens the BGS linewidth with the gain. Furthermore, we compare both methods, direct-detection BOTDA and coherent 


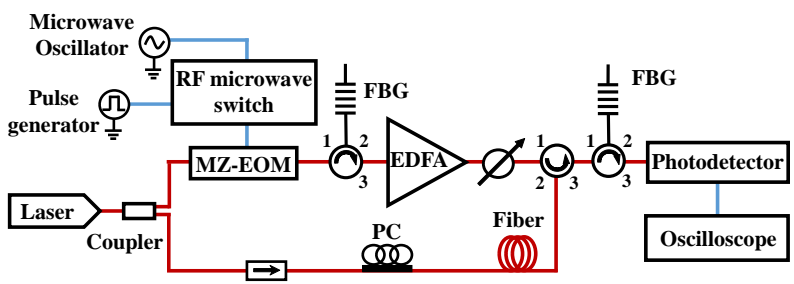

Fig. 1. Experimental setup for the conventional BOTDA system.

BOTDA, in terms of the amount of spectrum shape change with increasing Brillouin gain.

The paper is organized as follows. We first describe in section II the experimental study of the gain dependence of Brillouin gain linewidth in conventional direct-detection BOTDA sensors. This is done in order to complete the study in [7], providing results for additional pump pulse durations and powers, which let us to thoroughly compare the influence of Brilloiun gain in conventional direct-detection BOTDA sensors and in coherent-detection BOTDA sensors. Then, section III introduces the detailed analysis, theoretical and experimental, of the RF phase-shift spectrum shape dependence on Brillouin gain in coherent BOTDA.

\section{VERIFICATION OF GAIN DEPENDENCE OF BRILLOUIN LINEWIDTH IN DIRECT-DETECTION BOTDA}

In order to measure the gain dependence of the Brillouin linewidth in direct-detection BOTDA sensors, we deployed the experimental setup depicted in Fig. 1 [10]. A distributed feedback laser (DFB) laser is used as the optical source. Then, in the upper branch of the setup, optical pump pulses are formed using a Mach-Zehnder electro-optic modulator (MZ-EOM) biased at minimum and driven by microwave pulses. One of the two pump sidebands generated is filtered with a fiber Bragg gratting (FBG) and amplified by a high-power erbium doped fiber amplifier (EDFA). Then, an optical attenuator controls the pump power injected to the fiber, which can reach a peak value of $4 \mathrm{~W}$. The peak pump pulse power has been measured in the time domain using a calibrated photodetector. In the lower branch, the DFB output is directly used as probe wave using an optical coupler. This probe wave is injected into the fiber after its polarization state is adjusted to optimize the Brillouin interaction along 30-m of single-mode fiber (SMF-28). The resultant probe wave is first filtered in order to suppress the undesired spectral components, detected in a photo-receiver and captured in a digital oscilloscope.

Fig. 2 and Fig. 3 show the Brillouin linewidth broadening for 11-ns and 30-ns pump pulses when gain is risen up to $1.8 \mathrm{~dB}$ and $6.7 \mathrm{~dB}$, respectively. The spectra have been measured by sweeping the frequency separation between pump and probe in the setup of Fig. 1. The measured probe power has been normalized to the probe power with no Brillouin interaction and then the natural logarithm of the result has been computed to obtain the logarithmic gain. Notice that, as expected, the linewidth for the 11-ns pulses is larger than for 30-ns pulses as the former has a duration closer to the acoustic phonon lifetime. In these two cases, a full width at

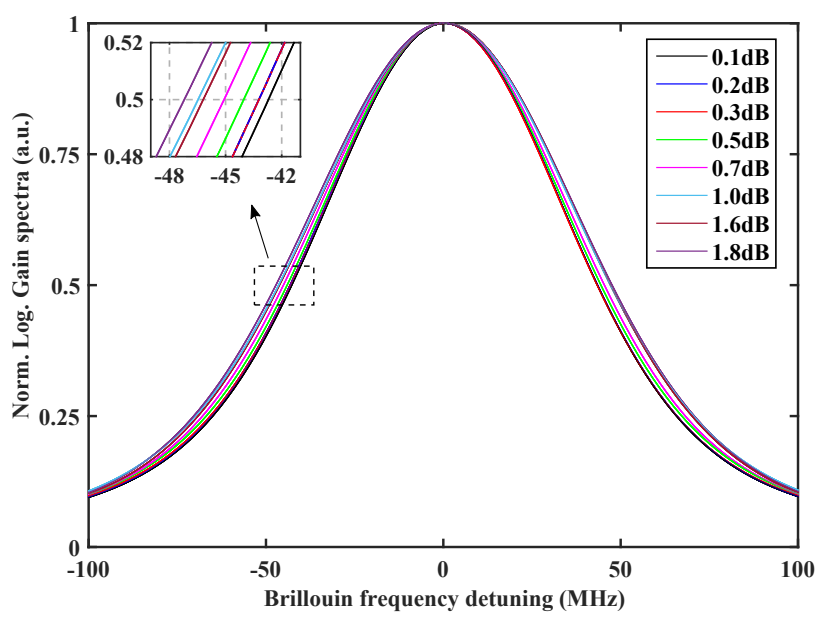

Fig. 2. Experimental Brillouin gain spectra for 11-ns pulse duration when gain increases from 0.1 to $1.8 \mathrm{~dB}$.

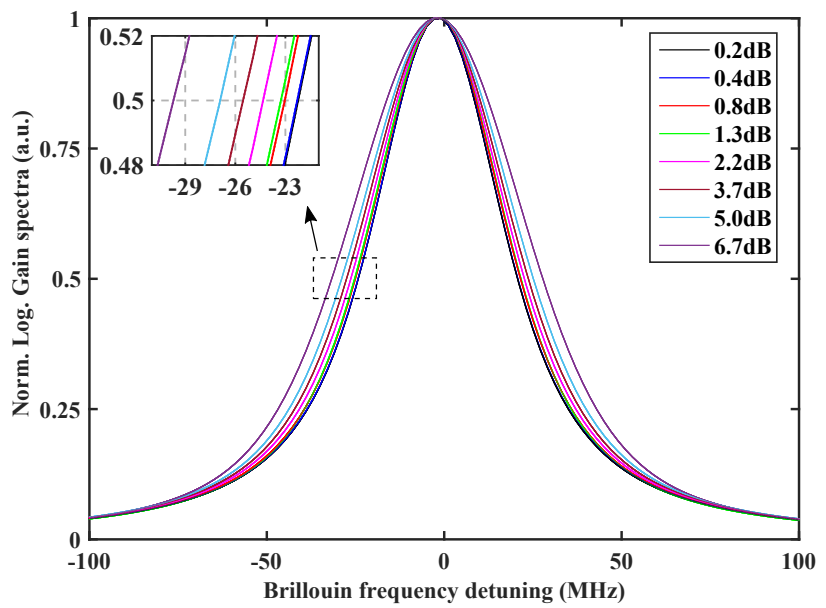

Fig. 3. Experimental Brillouin gain spectra for 30-ns pulse duration when gain increases from 0.2 to $6.7 \mathrm{~dB}$.

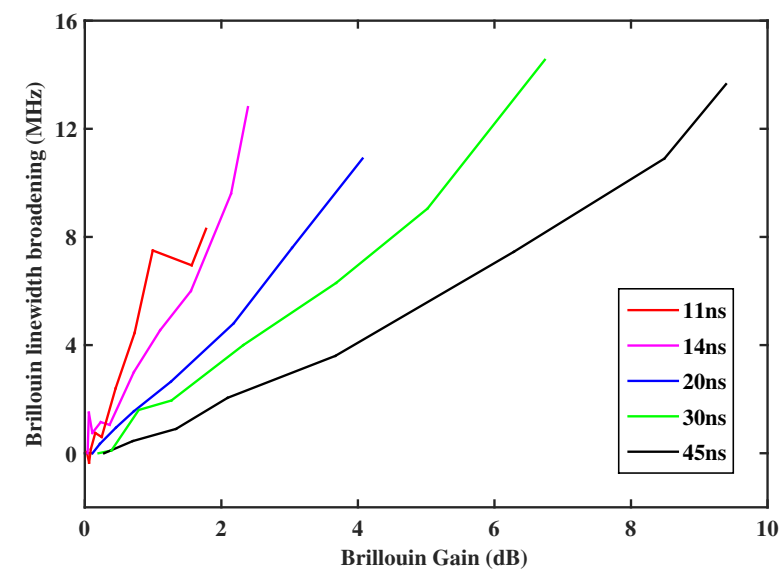

Fig. 4. Brillouin linewidth broadening for different pulse lengths when gain is risen.

half maximum (FWHM) linewidth of $45 \mathrm{MHz}$ and $86 \mathrm{MHz}$ has been measured for the 30-ns and 11-ns pulses, respectively. Moreover, the Brillouin gain spectra linewidth obtained for 


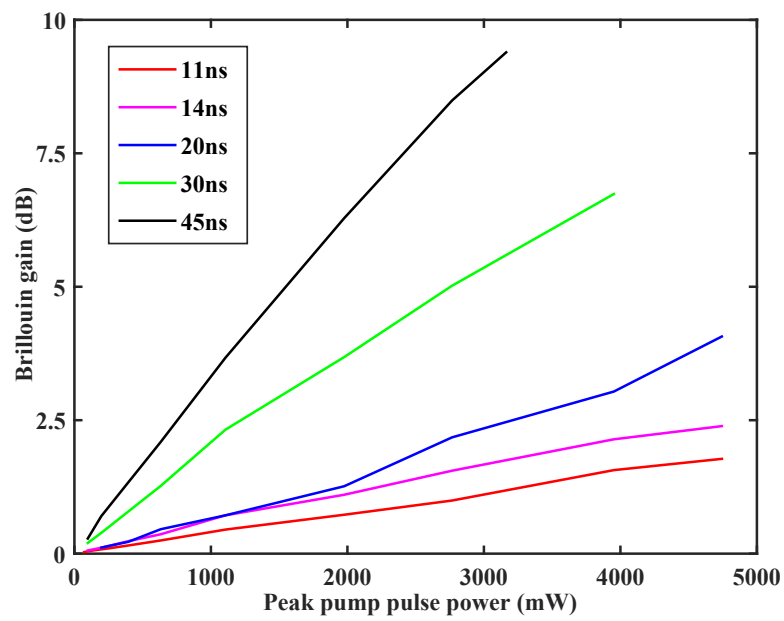

Fig. 5. Brillouin gain generated in relation to the peak pulse power injected to the fiber for different pulse lengths.

30-ns pulses tends to a Lorentzian profile, while that for 11-ns pulses are more similar to a Voigt or Pseudo-Voigt profile. This is a well known effect since the effective Brillouin gain spectrum results from the convolution of the intrinsic Brillouin spectrum and the pump pulse spectrum. Apart from this broadening effect, it has been also observed that the spectra in Fig. 2 and Fig. 3 progressively broadens for increasing gain. The observed FWHM broadening rate or gradient is also different for both pulses: $4 \mathrm{MHz} / \mathrm{dB}$ for 11-ns pulses and 2.2 $\mathrm{MHz} / \mathrm{dB}$ for 30 -ns pulses.

This same broadening effect has been analyzed for different pulse durations, as it is summarized in Fig. 4. It is clearly shown that the linewidth broadening suffered by large pulses is less significant than by short pulses. In general terms, the linewidth broadening observed agrees well with the results presented previously [7], hence independently demonstrating the existence of this effect. Moreover, the demonstration is performed with a different type of BOTDA experimental setup, hence possible origins of the linewidth broadening effect due to unintended experimental conditions are less probable.

Fig. 5 displays the dependence of the Brillouin gain on the pump pulse power for different duration of the pulses. As it is expected, longer pulses require less power to generate the same gain than shorter pulses because the interaction length is larger.

\section{GAIN DEPENDENCE IN COHERENT BOTDA}

Once the Brillouin linewidth dependence on gain has been confirmed in conventional direct-detection BOTDA, we turned our attention to investigate the influence that this effect could have in the coherent self-heterodyne BOTDA with phase-modulated probe wave. However, even though some influence was expected, its nature was not clear in advance because in coherent BOTDA the detection process involves the complex interplay of several waves. Therefore, in this section, we start by describing the fundamentals of coherent BOTDA and introducing the mathematical expressions for the detected signal. Then, we discuss the experimental and analytical results of the changes of the spectral shape for these sensors with increasing gain.

\section{A. Fundamentals}

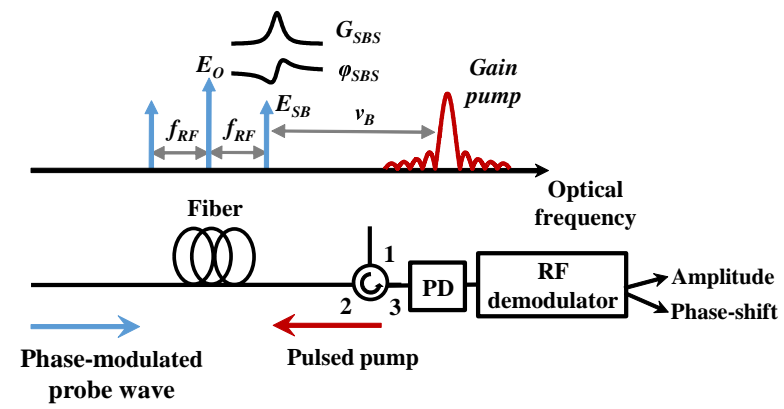

Fig. 6. Schematic representation of SBS interation for a coherent BOTDA system based on a phase-modulated probe wave.

The fundamentals of coherent BOTDA are schematically depicted in Fig. 6 [3]. A phase-modulated probe wave is injected in one end of an optical fiber while a pump pulse is introduced at the other end. This probe wave interacts with the pump pulse via stimulated Brillouin scattering. If SBS interaction only affects the upper sideband of the phase-modulated wave, the probe wave power detected in the photo-detector at the modulation frequency, $f_{R F}$, as a result of the interaction with the pulse at a particular location, $z$, can be expressed as [3]:

$$
\begin{array}{r}
\left.P(t)\right|_{f_{R F}}=2 E_{0} E_{S B}\left[G_{S B S} \cos \left(2 \pi f_{R F} t+\varphi_{S B S}\right)\right. \\
\left.-\cos \left(2 \pi f_{R F} t\right)\right]
\end{array}
$$

where $G_{S B S}$ and $\varphi_{S B S}$ are the amplitude and phase-shift of the Brillouin gain spectrum, and $E_{S B}$ and $E_{0}$ are the complex amplitude of the carrier and sidebands of the phase-modulated probe wave. In the case of a Lorentzian gain spectrum, $G_{S B S}$ and $\varphi_{S B S}$ are given by:

$$
\begin{gathered}
G_{S B S}=\exp \left(g_{0} \frac{\Delta \nu_{B}^{2}}{\Delta \nu_{B}^{2}+4 \Delta \nu^{2}}\right) \\
\varphi_{S B S}=-g_{0} \frac{2 \Delta \nu_{B} \Delta \nu}{\Delta \nu_{B}^{2}+4 \Delta \nu^{2}}
\end{gathered}
$$

where $g_{0}$, is the peak of the Brillouin gain spectrum, $\Delta \nu_{B}$, the Brillouin linewidth and, $\Delta \nu$, the detuning of the probe wave frequency from the maximum of the Brillouin interaction at the BFS.

Note that (1) represents the interference of two terms resulting from the beating between the optical carrier and each of the sidebands upon detection. Just one of the sidebands, and hence one of the terms, is affected by the Brillouin spectrum. Without Brillouin gain, both terms interfere destructively and no signal is detected. This is schematically depicted in the phasorial diagram in Fig. 7, where the two blue phasors represent the two signals that result from the beating of each 


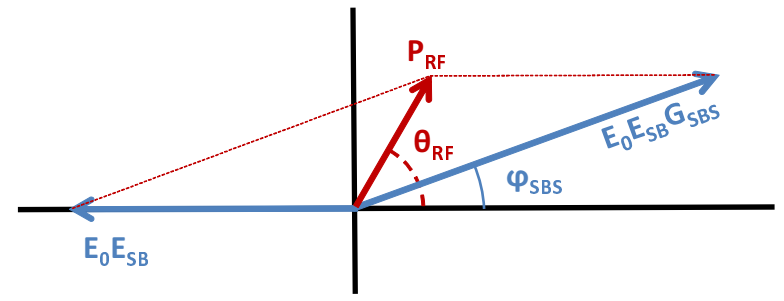

Fig. 7. Phasorial representation of SBS interaction for a coherent BOTDA system based on a phase-modulated probe wave.

of the two sidebands with the carrier and the red phasor is the resulting RF signal detected at the photo-receiver.

If we assume small gain and Lorentzian Brillouin gain, the expression in (1) can be approximated as:

$$
\begin{aligned}
\left.P(t)\right|_{f_{R F}} & \approx \frac{2 E_{0} E_{S B} g_{0} \Delta \nu_{B}}{\sqrt{\Delta \nu_{B}^{2}+(2 \Delta \nu)^{2}}} \\
& \cdot \cos \left[2 \pi f_{R F} t-\arctan \left(2 \frac{\Delta \nu}{\Delta \nu_{B}}\right)\right]
\end{aligned}
$$

Note that, under this approximation, the RF phase-shift of the detected signal, $\theta_{R F}$, is independent of the peak Brillouin gain. However, it is necessary to further investigate the behavior of the RF phase-shift spectrum shape for large gain, so an exact analytical expression for $\theta_{R F}$ has been derived from (1) with the help of the phasorial diagram in Fig. 7:

$$
\theta_{R F}=\arctan \left(\frac{G_{S B S} \cdot \sin \left(\varphi_{S B S}\right)}{G_{S B S} \cdot \cos \left(\varphi_{S B S}\right)-1}\right)
$$

And replacing (2) and (3) into (5), the resultant expression for $\theta_{R F}$ is shown in (6). Notice that this expression is obtained assuming that the Brillouin gain spectrum is Lorentzian, so it is only valid for pulses with a duration significantly longer than the acoustic phonon lifetime.

\section{B. Experimental setup}

Fig. 8 depicts the experimental setup deployed for the coherent BOTDA sensor, which is basically the same as the conventional setup described previously but adding a phase-modulator in the lower branch and a RF demodulator after detection. The phase-modulated probe wave generated is injected into the fiber and interacts with the pump pulse generating the Brillouin amplification in the fiber. Then, the probe wave is directed to the receiver where a coherent self-heterodyne detection is performed, the resultant RF-signal is processed with a RF-demodulator to obtain baseband signals that are captured in the oscilloscope.

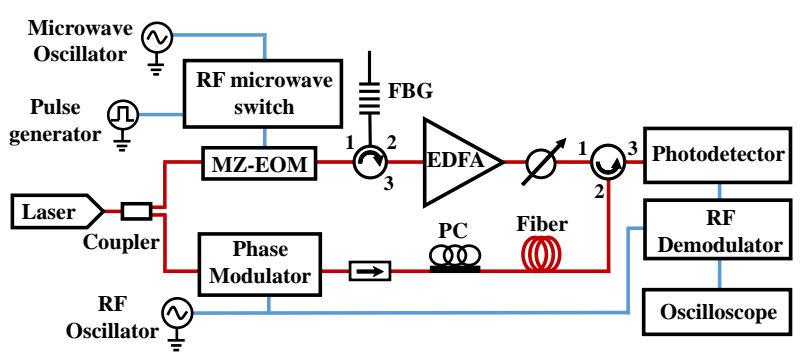

Fig. 8. Experimental setup for the coherent BOTDA system.

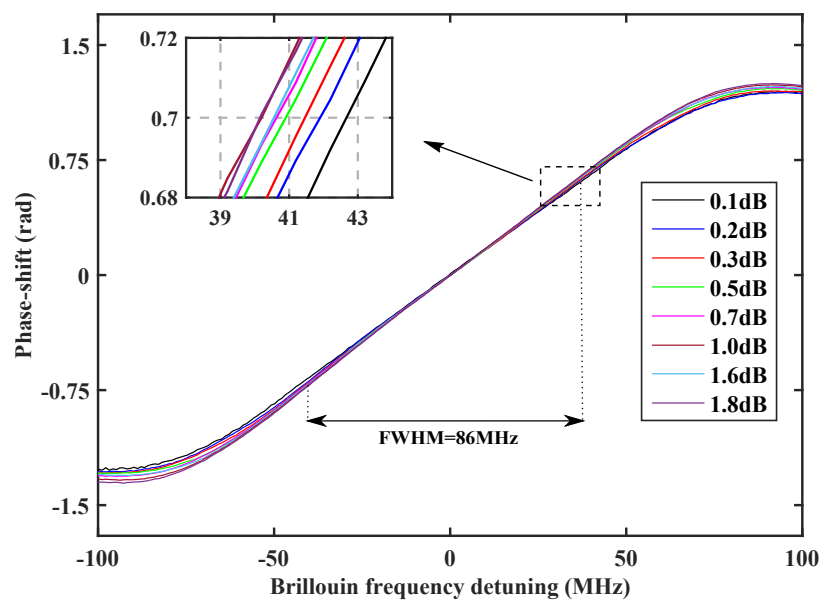

Fig. 9. Experimental RF phase-shift spectra for 11-ns pulse when gain increases from 0.1 to $1.8 \mathrm{~dB}$.

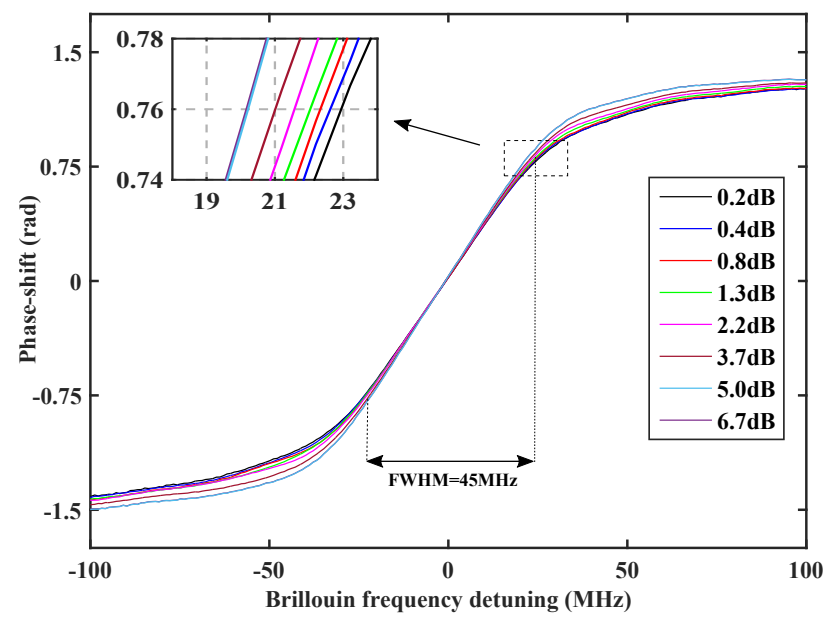

Fig. 10. Experimental RF phase-shift spectra for 30-ns pulse when gain increases from 0.2 to $6.7 \mathrm{~dB}$.

\section{Experimental results}

Experimental RF phase-shift spectra for increasing Brillouin gain, measured for 11-ns and 30-ns pulses, respectively, are shown in Fig. 9 and Fig. 10. In these figures,

$$
\theta_{R F}=\arctan \left(\frac{\exp \left(g_{0} \frac{\Delta \nu_{B}^{2}}{\Delta \nu_{B}^{2}+4 \Delta \nu^{2}}\right) \cdot \sin \left(-g_{0} \frac{2 \Delta \nu_{B} \Delta \nu}{\Delta \nu_{B}^{2}+4 \Delta \nu^{2}}\right)}{\exp \left(g_{0} \frac{\Delta \nu_{B}^{2}}{\Delta \nu_{B}^{2}+4 \Delta \nu^{2}}\right) \cdot \cos \left(-g_{0} \frac{2 \Delta \nu_{B} \Delta \nu}{\Delta \nu_{B}^{2}+4 \Delta \nu^{2}}\right)-1}\right)
$$


the RF phase-shift spectrum is wider for 11-ns pulses than the one for 30-ns pulses, which, similarly to conventional direct-detection BOTDA, is due to the broadening effect of the measured Brillouin spectrum resulting from the convolution of the pulse spectrum and the intrinsic Brillouin gain. In both cases, a gain dependence of the RF phase-shift spectrum shape is observed. However, the resultant measured spectra get narrower when the gain is increased, contrary to conventional BOTDA systems response, where the linewidth broadens with increasing gain.

As for the phase-shift spectra there is no clear metrics to characterize its shape, such as the FWHM for amplitude responses, we evaluated the narrowing taking as reference the RF phase-shift values that correspond to the frequencies of the FWHM of the amplitude spectrum for small gain. Then, we calculate the frequency difference between the initial FWHM frequencies and the new frequencies at which are obtained the RF phase-shift reference values when Brillouin gain is risen. The frequency region defined by the FWHM of the Brillouin gain amplitude spectrum matches quite well with the linear region of the RF phase-shift spectrum curves as it can be seen in Fig. 9 and Fig. 10. Therefore, the FWHM frequencies are a good estimator of the BFS measurement range for a coherent-detection BOTDA setup. These limits are marked in the figures with dashed-straight lines. These detuning frequencies have been calculated from the Brillouin amplitude spectra obtained in the conventional BOTDA system study presented in Section II. Some examples of the narrowing effect for the RF phase-shift spectrum in coherent BOTDA systems are shown in Fig. 9 and Fig. 10, where the 11-ns pulse narrows $2 \mathrm{MHz}$ when the gain is increased to $1 \mathrm{~dB}$, while for a 30-ns pulse, it narrows by $0.7 \mathrm{MHz}$.

These measurements have been repeated for different pulse durations (11 ns, 14 ns, $20 \mathrm{~ns}, 30 \mathrm{~ns}$ and $45 \mathrm{~ns}$ ) and the narrowing obtained as a function of the Brillouin gain is displayed in Fig. 11. Again, the general trend is that the narrowing inversely depends on the pulse duration. Furthermore, the comparison of Fig. 4 and Fig. 11 demonstrate that the gain influence on RF phase-shift spectrum measured in coherent BOTDA sensors is much smaller than on the Brillouin gain spectrum measured in conventional direct detection BOTDA sensors.

Notice than in our preliminary work in [3] a moderate maximum peak power of $26 \mathrm{dBm}$ was deployed, obtaining $0.5 \mathrm{~dB}$ of peak Brillouin gain, which, extrapolating from the data in Fig. 11, translates to a narrowing of the RF phase-shift of the order of $1 \mathrm{MHz}$. This narrowing was comparable to the measurement uncertainty of those experiments, and hence it was not visible in the results. In order to observe narrowing we need to deploy higher power pump pulses. These are feasible just in short lengths of fiber, where nonlinear effects are negligible.

Another parameter that can be used to quantify the modification of the RF phase-shift spectrum for increasing gain is the change of the slope of the linear region of the spectrum. Moreover, this parameter is directly linked to dynamic measurements that rely on this slope to translated BFS changes into modification of the measured $\theta_{R F}$. Fig. 12 depicts the slope change at the center of the spectra as a function of

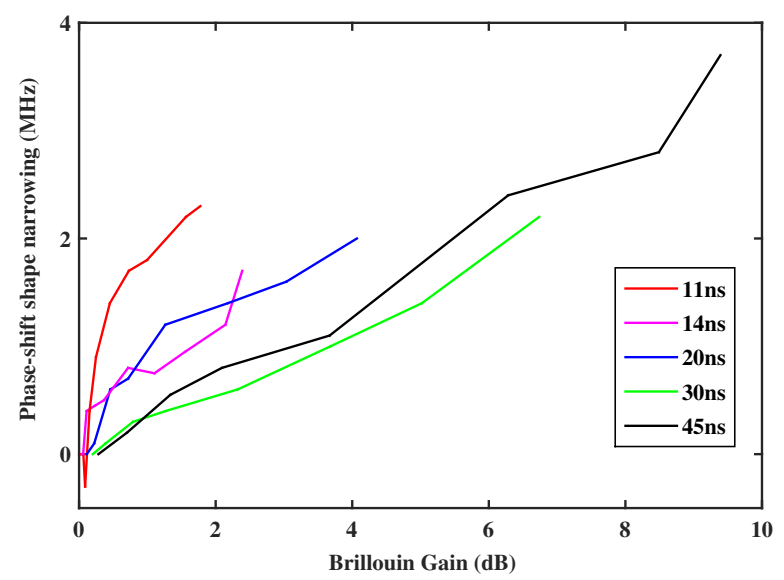

Fig. 11. RF phase-shift shape narrowing for different pulse lenghts when gain is risen.

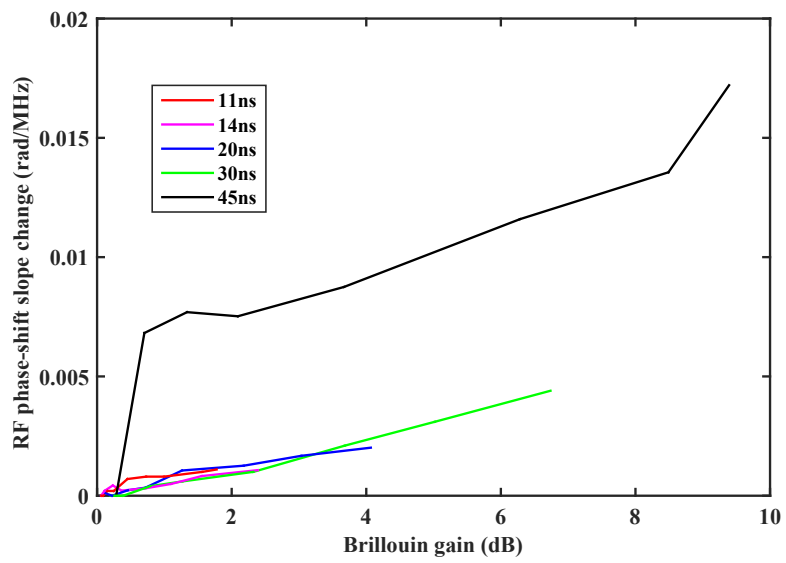

Fig. 12. Experimental RF phase-shift spectrum slope change due to Brillouin gain.

peak Brillouin gain for several pulse durations. It is noticeable that the dependence of the slope changes on the logarithmic gain is approximately linear for every pulse duration evaluated. Notice that, the RF phase-shift slope change curve using 45-ns pulse duration differs from the rest of the curves due to the interplay of the narrowing and the broadening effects that will be explained in the following section. This compensation is different using long pulse durations (such as $45 \mathrm{~ns}$ ) since the broadening effect is less significant as depicted in Fig. 4., and hence, both effects do not compensate as much as using short pulse durations.

\section{Calculated results and discussion}

The reason for the unexpected RF phase-shift spectrum narrowing (instead of broadening) on coherent BOTDA systems was investigated using the model presented in (6), which is valid for relatively long duration pulses whose spectra can be approximated by a Lorentzian function. Thus, we first evaluated the expression of $\theta_{R F}$ for different peak Brillouin gains assuming a typical $\Delta \nu_{B}$ value of $33 \mathrm{MHz}$. As it can be observed in Fig. 13(a), the narrowing effect of the 


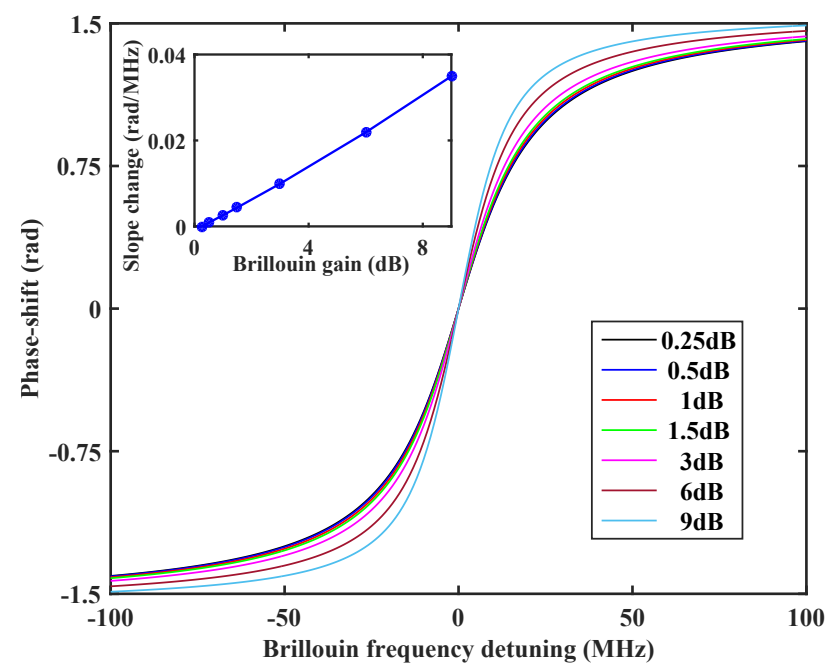

(a)

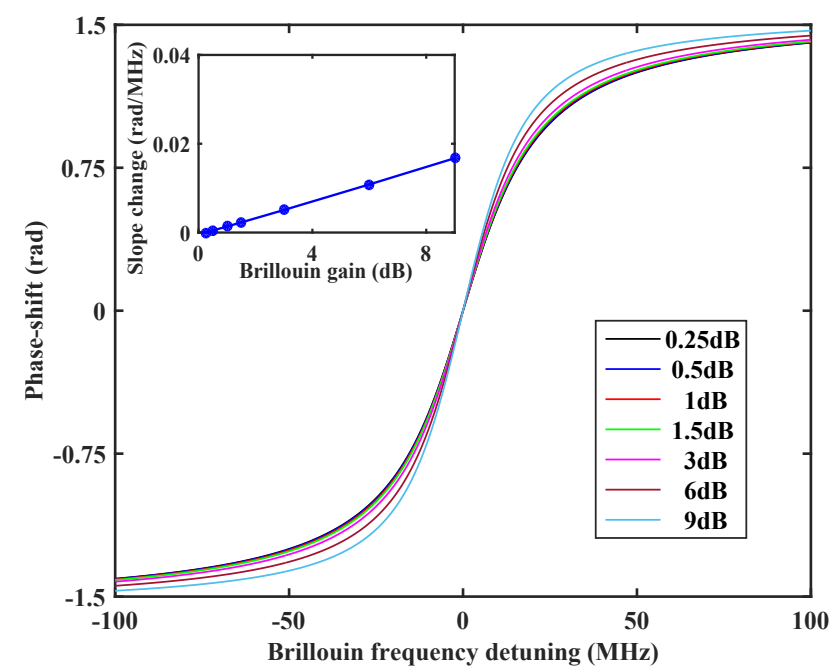

(b)

Fig. 13. Calculated phase-shift narrowing for long pulses using expression (6): (a) under the influence of gain increment; (b) under the influence of gain increment and Brillouin linewidth broadening.

RF phase-shift is also noticed and indeed demonstrated mathematically. The inset of Fig. 13(a) depicts the dependence of the slope of $\theta_{R F}$ on logarithmic gain, which also follows the linear trend observed in the experimental measurements. However, previous calculations assume a constant $\Delta \nu_{B}$ independent of gain, which the results in section II show that is not correct. Therefore, we completed the model in (6) by incorporating the dependence of $\Delta \nu_{B}$ on gain. This can be done by taking advantage of the following analytical expression for this dependence, which was derived in [8] and [7]:

$$
\Delta \nu_{B} \approx \frac{1}{2 \pi \tau_{A}}+\frac{3 g_{0}}{4 \pi T}
$$

where $\tau_{p}$ is the phonon lifetime, $\mathrm{T}$ is the time duration of the pulse employed for each measurement and $g_{0}$ is the maximum peak Brillouin gain given in $[\mathrm{dB}]$.

Fig. 13(b) depicts the reevaluation of previous calculations for the dependence of $\Delta \nu_{B}$ on gain, but this time taking into account the linewidth broadening given by (7). A reduction of the narrowing effect and of the change of the slope of $\Delta \nu_{B}$ with gain (inset of the figure) is clearly noticeable due to the counteracting influence of the linewidth broadening effect on the dependence of the RF phase-shift on the gain. Notice also that the slope change with gain depicted in the inset of the figure maintains its linearity but with smaller total change. Moreover, these calculated slope changes agree well with the experimental results for 45-ns pulses presented in Fig. 12.

The natural trend for the RF phase-shift spectra to narrow for increasing gain can be explained using a phasorial representation of the expression depicted in (1). This equation conveys the fact that the detection of the probe wave is the result of the interference of two RF signals, one coming from the beating of the optical carrier with the lower sideband, and the other coming from the beating of the optical carrier and the upper sideband of the phase-modulated probe wave. Without Brillouin gain, both RF signals have opposite phase and equal amplitude and hence they destructively interfere.
On the contrary, when there is Brillouin interaction, one of the RF signals changes its amplitude and phase. Fig. 14 represents the two interfering RF signal by two phasors (in blue). The detected RF signal is shown as another phasor (in red) that is the result of the addition of these two interfering phasors. The phase of this resultant phasor, $\theta_{R F}$, is the RF phase-shift that is measured in the coherent BOTDA sensor. Two examples of detected signal for increasing gain are depicted. Notice that, when Brillouin gain is increased, the phasor affected by Brillouin interaction increments its amplitude (Brillouin gain, $G_{S B S}$ ) and phase (Brillouin phase-shift, $\varphi_{S B S}$ ), leading to the blue dashed-line phasor instead of the blue solid-line phasor. The resultant red dashed-line phasor has a larger $\theta_{R F}$ than the lower-gain red solid-line phasor. If we translate this $\theta_{R F}$ behavior to one particular RF phase-shift spectrum curve, it means that the RF phase-shift value detected for a particular detuning frequency grows with gain, which implies that the RF phase-shift spectrum slope increases, hence, the spectrum shape becomes narrower with gain, as it has been observed experimentally and through previous calculations.

As it was mentioned before, the model in (6) is valid for pulses with duration longer than the acoustic lifetime. For shorter pulses the Brillouin gain spectra is no longer Lorentzian and there is not a close-form solution. In order

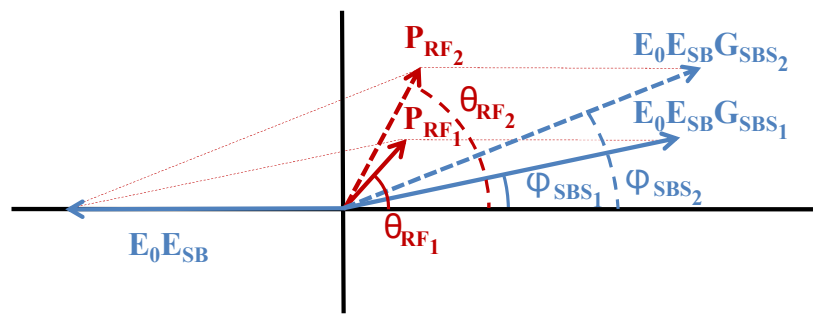

Fig. 14. Phase-modulated probe wave phasorial representation on a coherent BOTDA system. 


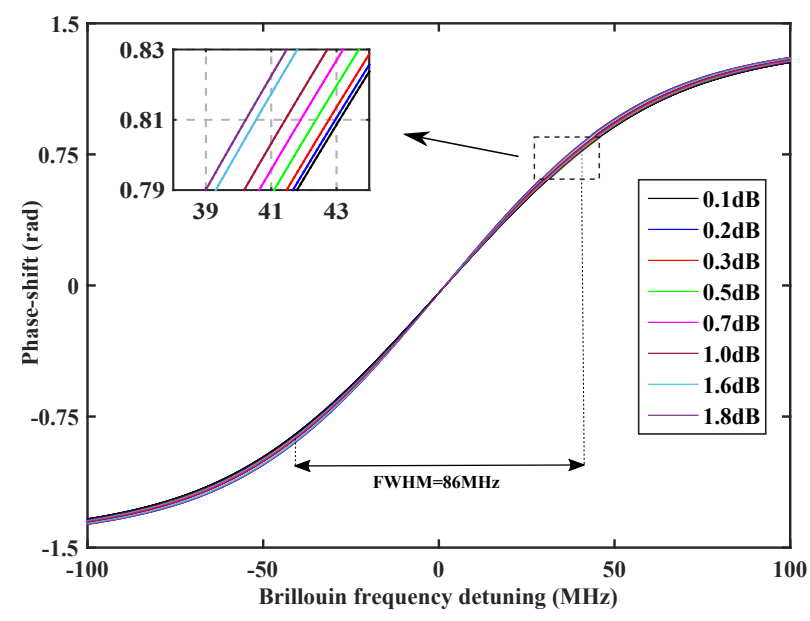

(a)

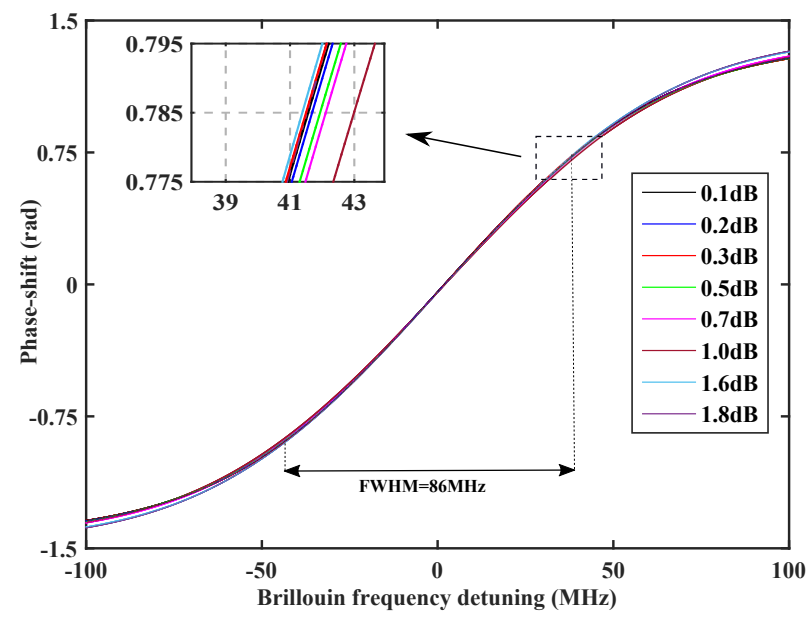

(b)

Fig. 15. Calculated phase-shift narrowing for 11-ns pulses considering: (a) only gain increment; (b) gain increment and Brillouin linewidth broadening.

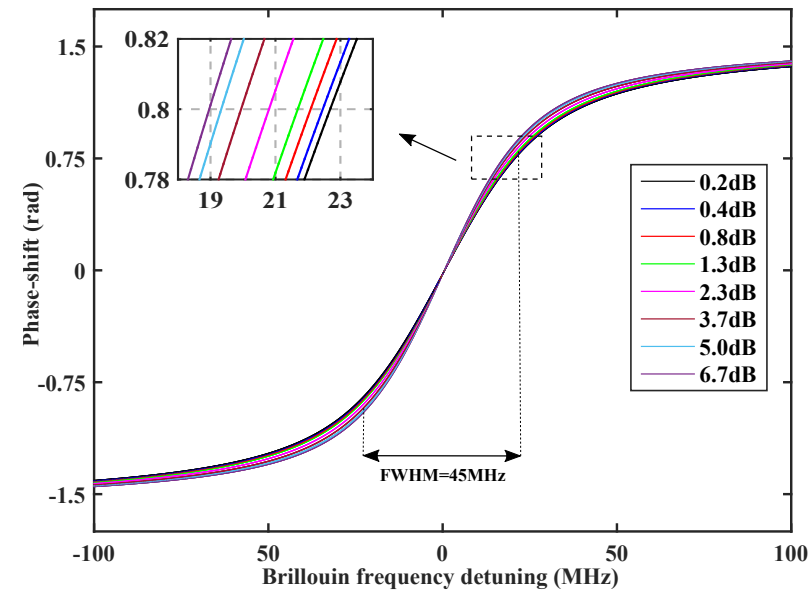

(a)

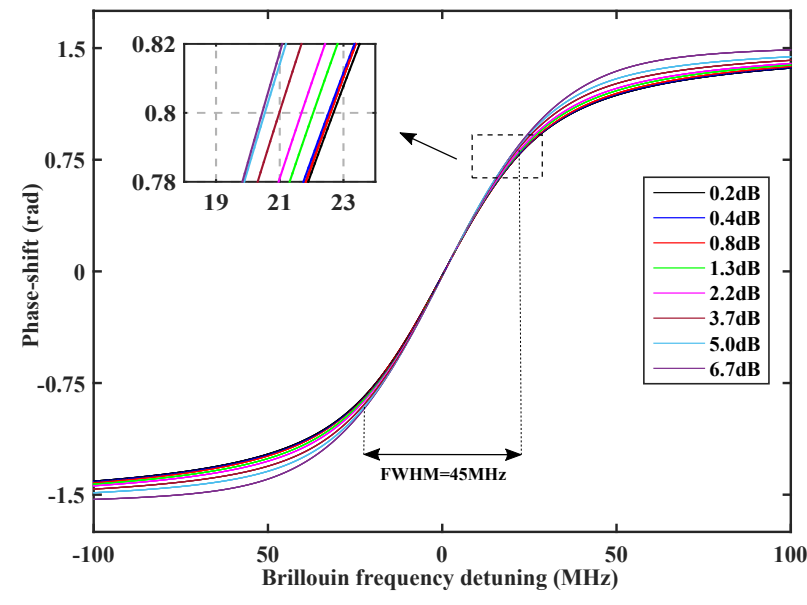

(b)

Fig. 16. Calculated phase-shift narrowing for 30-ns pulses considering: (a) only gain increment; (b) gain increment and Brillouin linewidth broadening.

to deal with shorter pulses we made the calculations using the general model in (1) but modeling the gain as the convolution of the pulse spectrum (assumed to be Gaussian) with the intrinsic Brillouin gain spectrum (Lorentzian). The resultant gain spectra follows a Faddeva profile [11], which displayed a better fit to the gain spectra measured in section II. In fact, the parameters needed for the model calculations were obtained by fitting the Faddeva profile to the experimental results for the Brillouin gain spectrum presented in Section II. Those parameters were the Lorentzian and Gaussian linewidth and the gain as a function of the pump pulse power for each pulse duration.

Fig. 15(a) and Fig. 16(a) show the calculated RF phase-shift spectrum as a function of gain for 11-ns and 30-ns pulses. Notice that there is a narrowing of the spectra for increasing gain. Again, these first calculations (Fig. 15(a) and Fig. 16(a)) were performed assuming that there were no gain dependence of the Brillouin linewidth, i.e., the width parameters of the Brillouin gain spectrum (Lorentzian and Gaussian linewidths) were fixed to those observed for small gain and just the peak gain was modified.

Therefore, the observed change in the shape (narrowing)

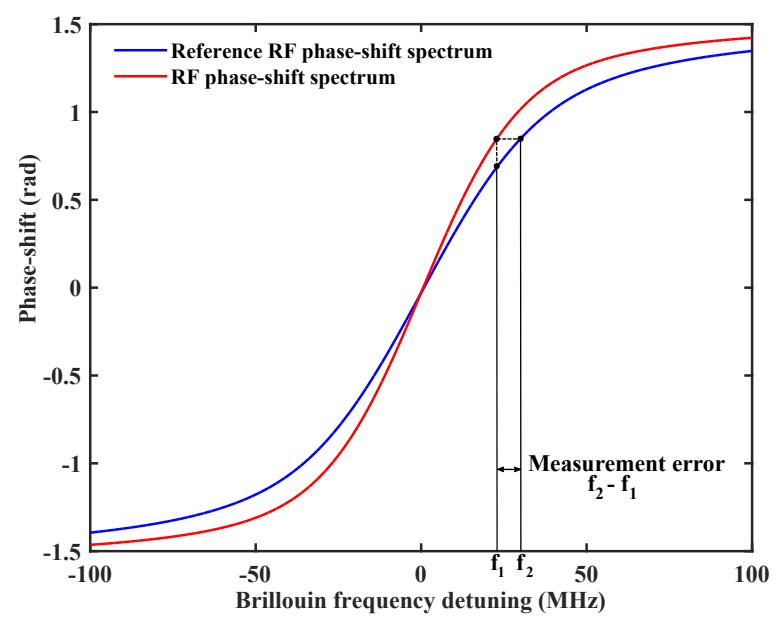

Fig. 17. Measurement error calculation example. 
in these spectra, is just due to the nature of the interference between detected waves that is conveyed by (1), and has nothing to do with the linewidth broadening effect introduced previously. However, the narrowing obtained in Fig. 15(a) and Fig. 16(a) is found to be larger than the actual narrowing measured in the experimental results of Fig. 9 and Fig. 10. This difference in the amount of phase-shift spectrum narrowing is due to the fact that Brillouin linewidth broadening was not taken into account. Thus, we again repeated the calculations, but this time incorporating the Brillouin linewidth broadening with increasing gain. These new results, which are shown in Fig. 15(b) and Fig. 16(b), highlight a reduced narrowing of the spectra, which agrees well with the results obtained in the experiments.

Therefore, we can conclude that in coherent BOTDA the natural trend to narrow of the RF phase-shift spectra for increasing gain is compensated to some extend by the linewidth broadening effect. This is the reason why the gain dependence of shape of the spectra is less important in these systems than in conventional BOTDA systems.

\section{E. Measurement error originated by the gain dependence of the RF phase-shift in coherent BOTDA sensors}

Finally, we studied the consequences that the gain dependence of the RF phase-shift spectra has for coherent BOTDA sensors in dynamic operation. An initial calibration is required to calculate the measuring error for this type of dynamic sensor. This is because the measured $\theta_{R F}$ will be directly translated to BFS by using the $\theta_{R F}$ versus frequency detuning (RF phase-shift spectrum) calibration curve performed initially. The calibration of the RF phase-shift spectra may be performed at either low pump pulse power (small Brillouin gain) or high pump pulse power, (high Brillouin gain). For both calibration options, it is necessary to characterize the error that the change in shape (narrowing) for large Brillouin gains brings to the measured dynamic BFS. Fig. 17 depicts schematically how the error as a function of detuning frequency and Brillouin gain is calculated for given measurement conditions. The blue trace is the reference calibrated RF phase-shift spectrum, and red one is the measured RF phase-shift curve obtained when the Brillouin gain is increased. For a given measured $\theta_{R F}$, there is a mismatch between the corresponding $\Delta \nu$ value that the two spectra provide, $f_{1}$ and $f_{2}$. The error is calculated simply by subtracting these two values of $\Delta \nu$.

Therefore, the error will be dependent on the detuning induced by the change in BFS. Notice that, there is very small error for small BFS change because the narrowing of the RF spectrum close to $\Delta \nu=0$ is very small. The error grows as the frequency detuning between the Brillouin frequency shift and the probe wave increases. This is highlighted in Fig. 18 and Fig. 19, which depict the error for 11-ns and 30ns pulses due to the spectral narrowing when Brillouin gain is modified. This error is calculated from the experimentally measured RF phase-shift spectra assuming the low gain curve as the calibrated reference to obtain the measuring error. An alternative way to calculate the measuring error is using the RF phase-shift spectra slope change, however, this method is

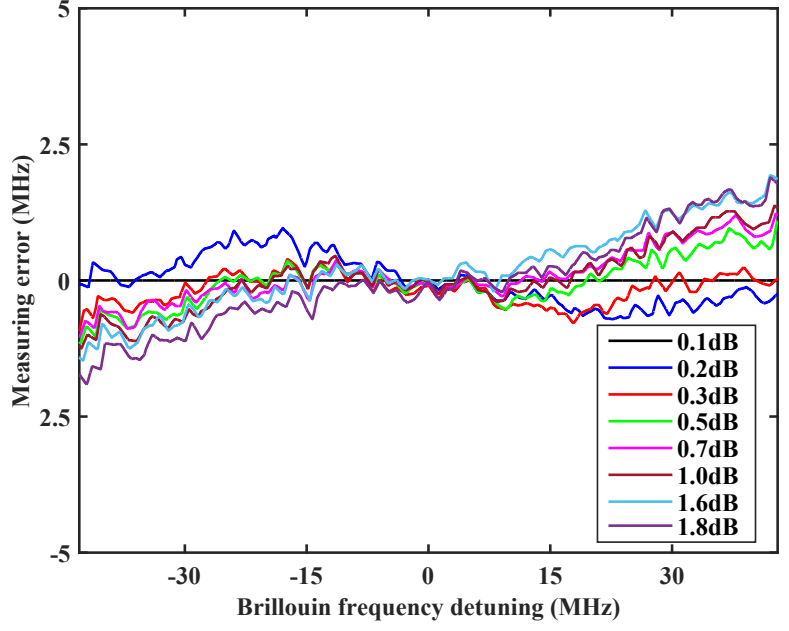

Fig. 18. Measuring error in coherent BOTDA system when Brillouin gain is modified and low gain spectrum is used for calibration with 11-ns pulses.

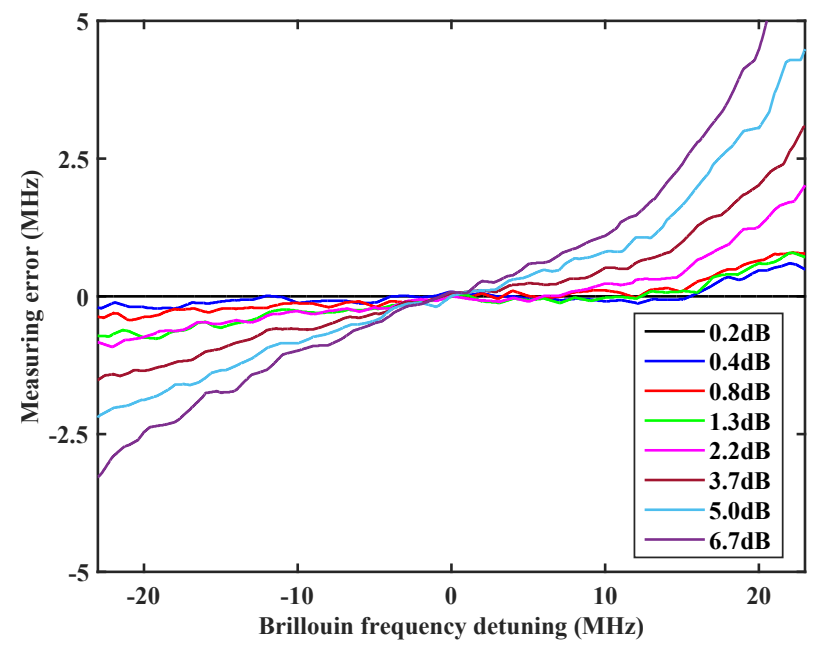

Fig. 19. Measuring error in coherent BOTDA system when Brillouin gain is modified and low gain spectrum is used for calibration with 30-ns pulses.

not as accurate as the one presented here for regions away from $\Delta \nu=0$ where the RF phase-shift spectra deviates from linearity.

The same measuring error calculations may be performed taking as reference curve the resultant RF phase-shift spectrum curve when maximum Brillouin gain is employed. Fig. ??(a) and Fig. ??(b) show the calculated error in that case, which is slightly more linear in the FWHM region. Furthermore, the error magnitude tends to be smaller due to the fact that the high gain curve has slightly larger linear region, so taking this as a reference for calibration improves the comparison to other curves for lower gains; hence, reducing the error.

These graphs can be used to calculate the trade-offs among the different parameters involved in a measurement, i.e., the desired BFS measurement range, maximum tolerable BFS error, and pump pulse power. For instance, in a particular situation where we assume that the low gain spectrum curve is taken for calibration and our working pump peak power is $20 \mathrm{dBm}$, a dynamic strain measurement is performed with $1-\mathrm{m}$ spatial resolution in which a maximum error of $20 \mu \epsilon(1 \mathrm{MHz}$ 
BFS) is admitted for a total measurement range of $1720 \mu \mathrm{\epsilon}$ (86 MHz). From Fig. 18, we find that the Brillouin gain should be below $0.5 \mathrm{~dB}$, which means that, looking at Fig. 5, the pump pulse power should be kept below $30.5 \mathrm{dBm}$, otherwise, the error committed with respect to the lower gain spectrum curve at the FWHM detuning frequency would be higher than $1 \mathrm{MHz}$. Another example would be a measurement with 3-m resolution, maximum error of $20 \mu \epsilon$ and measurement range of $760 \mu \epsilon(45 \mathrm{MHz})$. In this case, Fig. 19 shows that the Brillouin gain should be kept below $1.3 \mathrm{~dB}$ and, hence, the pump pulses below $28 \mathrm{dBm}$. Notice that, in general, larger peak powers can be used for shorter pulses.

Finally, we would like to stress that the pump pulse power limitation imposed by the RF phase-shift spectrum narrowing effect is only significant if it is below the threshold imposed by other nonlinear effects such as modulation instability (MI), as presented in Fig. 4 in [12]. For instance, for the given example measurements, when $30.5 \mathrm{dBm}$ peak power and 11-ns pulses are employed, MI will limit the maximum link lenghts to $2 \mathrm{~km}$ and, for $30-\mathrm{ns}$ lenght pulses and $28 \mathrm{dBm}$ pump peak power, MI will limit the maximum pump power from 5-km length [12]. Therefore, for measuring structures larger than 2-km length, MI will be the most limiting factor, instead of the error generated by the RF phase-shift spectrum shape change due to its dependence on the Brillouin gain in coherent BOTDA systems.

\section{CONCLUSIONS}

In summary, this work demonstrates that the RF phase-shift spectrum measured on coherent BOTDA sensors based on a phase-modulated probe wave is indeed gain dependent in large gain scenarios, but, in any case, this dependence is less significant than that found in conventional direct-detection BOTDA sensors for the gain spectrum broadening. The error that the gain dependence generates on the measuring system is dependent on the pump pulse duration and on the detuning frequency. From a practical point of view, it is found that this systematic error is not a serious limiting factor given the pump pulse power that are normally deployed in realistic application scenarios. Furthermore, this effect has significance only in very short fibers, because for longer lengths, nonlinear effects such as modulation instability, self-phase modulation or Raman scattering impose a more stringent limitation to the pump power that can be deployed.

\section{REFERENCES}

[1] R. Bernini, A. Minardo, and L. Zeni, "Dynamic strain measurement in optical fibers by stimulated Brillouin scattering," Opt. Lett., vol. 34, no. 17, pp. 2613-2615, Sep. 2009.

[2] Y. Peled, A. Motil, L. Yaron, and M. Tur, "Slope-assisted fast distributed sensing in optical fibers with arbitrary Brillouin profile," Opt. Exp., vol. 19, no. 21, pp. 19845-19854, Sep. 2011.

[3] J. Urricelqui, A. Zornoza, M. Sagues, and A. Loayssa, "Dynamic BOTDA measurements based on Brillouin phase-shift and RF demodulation," Opt. Exp., vol. 20, no. 24, pp. 26942-9, Nov. 2012.

[4] Y. Peled, A. Motil, and M. Tur, "Fast Brillouin optical time domain analysis for dynamic sensing," Opt. Exp., vol. 20, no. 8, pp. 8584-8591, Apr. 2012.

[5] A. Motil, O. Danon, Y. Peled, and M. Tur, "Pump-power-independent double slope-assisted distributed and fast Brillouin fiber-optic sensor," IEEE Photon. Technol. Lett., vol. 26, no. 8, pp. 797-800, Apr. 2014.

[6] D. Ba, B. Wang, D. Zhou, H. Zhang, Z. Lu, L. Chen, X. Bao, and Y. Dong, "Dynamic distributed Brillouin optical fiber sensing based on multislope analysis," in Proc. SPIE, vol. 9634, Sep. 2015.

[7] A. Motil, R. Hadar, I. Sovran, and M. Tur, "Gain dependence of the linewidth of Brillouin amplification in optical fibers," Opt. Exp., vol. 22, no. 22, pp. $27535-27541$, Nov. 2014.

[8] G. L. Keaton, M. J. Leonardo, M. W. Byer, and D. J. Richard, "Stimulated Brillouin scattering of pulses in optical fibers," Opt. Exp., vol. 22, no. 11, pp. 13351-65, 2014.

[9] A. Motil, I. Sovran, and M. Tur, "The effect of the gain dependency of the linewidth of Brillouin amplification on double slope-assisted dynamic sensing techniques," in Proc. SPIE, vol. 9634, Sep. 2015, pp. $8-11$.

[10] A. Zornoza, D. Olier, M. Sagues, and A. Loayssa, "Brillouin distributed sensor using RF shaping of pump pulses," Meas. Sci. Technol., vol. 21, no. 9, July 2010.

[11] G. P. M. Poppe and C. M. J. Wijers, "More efficient computation of the complex error function," ACM Transactions on Mathematical Software, vol. 16, no. 1, pp. 38-46, 1990.

[12] M. Alem, M. Soto, and L. Thévenaz, "Analytical model and experimental verification of the critical power for modulation instability in optical fibers," Opt. Exp., vol. 23, no. 23, pp. 29514-29532, Nov. 2015. 
(C) (C) 2016 IEEE. Personal use of this material is permitted. Permission from IEEE must be obtained for all other uses, in any current or future media, including reprinting/republishing this material for advertising or promotional purposes, creating new collective works, for resale or redistribution to servers or lists, or reuse of any copyrighted component of this work in other works. 